Thanks are due to Dr. J. K. Chowdhury, head of the Department of Chemistry, and Dr. B. Banerjee, senior research fellow, Bose Research Institute, Calcutta, for their encouragement and suggestions during this work. My thanks are also due to Dr. S. K. Mukherjee, University College of Science and Technology, Calcutta, for his criticism and suggestions.

Bose Institute, 93 Upper Circular Road,

$$
\text { D. P. Burma }
$$

Calcutta 9.

${ }^{1}$ Consden, R., Gordon, A. H., and Martin, A. J. P., Biochem. J., 38, 224 (1944).

${ }^{2}$ Wrilliams, R. J., and Kirby, H. M., Science, 10\%, 481 (1948).

\section{A Convenient 'Hanging-drop' Glass Electrode Unit}

A GLASS electrode unit developed in this laboratory has proved so generally useful that its brief description may be helpful to others. It combines the advantages of providing a robust and convenient assembly for general purposes, and of permitting precise measure. ment of $p \mathbf{H}$ with as little as $0.15 \mathrm{ml}$. of available solution if need be. The form of the unit (which

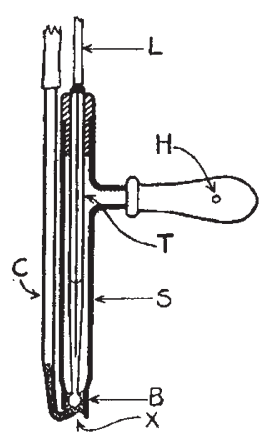
incorporates an effective stirring device) makes it suitable for electrometric titrations on volumes of solution as small as $0.4 \mathrm{ml}$.

The construction of the electrode assembly is shown in the diagram. The glass membrane is the bulb $B$, about $4 \cdot 8-5 \cdot 0$ $\mathrm{mm}$. diameter, blown on the end of the drawn-out tube $T$ which contains a sealed-in $N$ hydrochloric acid/silver chloride half-cell. It is a great advantage that the membrane consists of a type of glass permitting determinations of $p H$ in alkaline as well as acid solution.

The electrode is mounted within a glass sleeve $S$, of which the main upper portion is $8 \mathrm{~mm}$. internal diameter, and the short bottom portion about $6.2 \mathrm{~mm}$. A side tube near the top is fitted with a rubber teat; this is perforated by a small hole $H$ normally closed by the thumb on compressing. This provides a means of filling, washing, and also of stirring when the electrode is dipped into a small volume of liquid.

The bottom of the sleeve is open. A small notch ground in its bottom edge receives the bent tip of the potassium chloride bridge $C$, which is bound to the sleeve by rubber bands. The tip of $C$, including the bend, consists of a capillary with a sealed-in thread of asbestos fibres which terminates the salt bridge. The tube $C$ is connected above to a reservoir (not shown) of potassium chloride into which a small calomel half-cell is dipped, the connexion being made through a length of about $12 \mathrm{~cm}$. of flexible plastic tubing.

The potassium chloride reservoir, and the electrical lead $L$, are clamped on a suitable stand and the electrode assembly is allowed to dangle at a convenient height above bench-level.

The test solution $X$ surrounds the glass electrode $B$ and is held in place merely by capillarity. It is in contact with the tip of the potassium chloride bridge.

The general method of operation will be clear to any user, but the following may be mentioned:
(1) pointed pieces of filter paper may be used to drain the space around the electrode, for example, during washing; (2) to fill when minimal liquid is available, a drop placed at the bend in $C$ will enter the space around the electrode.

I wish to acknowledge the helpful co-operation of Messrs. Doran Instrument Co., Ltd., Stroud, Glos, who assisted by developing an appropriate glass electrode for this unit.

\section{Agricultural Research Council}

Charles S. Hanes

Unit of Plant Biochemistry,

Botany School, Cambridge. July 27.

\section{A Gravimetric Estimation of the Carboxylic Acid Group in Cellulose}

THE electrochemical and ion exchange properties of cellulose and other insoluble polysaccharides are largely determined by the content of - $\mathrm{COOH}$ groups, so that their precise estimation becomes a matter of some importance. Davidson ${ }^{1}$ has recently reexamined the various methods available for their determination, which are based either upon some form of acid-alkali titration, or upon the absorption of the basic dye methylene blue.

We consider most of the defects of these earlier methods are absent in the new method we now describe. The material is soaked in a saturated solution of uranium acetate in water $(c .0 \cdot 01 \mathrm{M}, p \mathrm{H} 6 \cdot 5)$ over-night, very thoroughly washed, oven-dried and weighed, ashed at $700^{\circ} \mathrm{C}$. and the ash weighed as $\mathrm{U}_{2} \mathrm{O}_{5}$. This procedure applied to commercial 'Cellophane' gave the following results:

$\begin{array}{cccc}\text { mgm. Ash/1 gm. 'Cellophane' } & 13 \cdot 56 & 13 \cdot 86 & 13 \cdot 97 \\ \text { Acid-washed blank } & 0 \cdot 1 & 0 \cdot 1 & 0.1 \\ \text { Mean COOH content milliequiv./100 gm. }=4 \cdot 60 . & \end{array}$

One of us (G.R.W.) has also estimated the uranium content by weighing in water before and after soaking in uranium acetate, using a hydrostatic balance. An average result of 4.71 milliequiv. $\mathrm{COOH} / 100 \mathrm{gm}$. was obtained.

The same material gave the following results by other methods:
Neale and Stringfellow's method (ref. 2)
Methylene blue absorption (ref. 1)
J. Farrar
S. M. NEALE
G. R. Williamson

College of Technology,

Manchester 1.

' Davidson, Shirley Inst. Mem., 22, 71 (1948).

${ }^{2}$ Neale and Stringfellow, Trans. Farad. Soc., 33, 881 (1937).

${ }^{3}$ Pacsu, J. Text. Research, 16, 163, 318, 390 (1946).

\section{William Nicol and Henry Clifton Sorby}

IN her short article on William Nicol and $\mathrm{H}$. C. Sorby, Mrs. Eyles states" that Nicol "seems to have confined himself to making sections of fossil wood, and did not make rock sections". Nicol stated in $1834^{2}$, however, that when he came to prepare sections of fossil plants he "had recourse to a process I had practised upwards of fifteen years ago, in preparing thin slices of the most fragile substances, as calcareous spar, in order to examine their effects on polarized light'. The lapidary G. Sanderson had made thin sections after fixing the specimen to a 